\title{
QUANDO GLI ATTRIBUTI TRAVALICANO IL SIGNUM. RIFLESSIONI SULL' IDENTITÀ VISUALE DEGLI DÈI A ROMA* \\ WHEN ATTRIBUTES GO BEYOND THE SIGNUM. REMARKS ON THE VISUAL IDENTITY OF THE GODS IN ROME
}

Ginevra Benedetti Università di Pisa / Université Toulouse JeAn Jaurès GINEVRABENEDETTI2404@GMAIL.COM

\section{Riassunto}

In questo lavoro ci si propone di analizzare, attraverso le pagine degli autori latini, la costruzione semiotica sottostante la rappresentazione visuale degli dèi nella cultura romana; ognuno di loro possedeva

\section{Abstract}

In this work we aim to analyze, through the pages of the Latin authors, the semiotic construction underneath the visual representation of the gods in the Roman culture; each of them possessed some attribute or 
infatti qualche attributo o combinazione di attributi in grado di identificarli con maggiore o minore certezza, ciò che gli autori antichi definivano insignia, dei "segni speciali” che guidavano l'interpretazione / identificazione di un signum. In particolare, saranno presi in esame alcuni oggetti concreti impiegati dalla cultura romana per costruire immagini divine nella loro funzione di attributi dotati di una specifica identità semiotica. A Roma vi erano altresì casi specifici in cui il potere dell' insigne risultava traboccante; la marca di riconoscibilità, in questi casi, era identificata con la totalità dell' immagine, così come l' immagine totale era ridotta e identificata con l'estensione della marca di riconoscimento: l' insigne, in questo caso, costituiva l' immagine. Questo ci porterà dunque a discutere i metodi di costruzione, adattamento, prestito e scarto delle immagini divine tra i politeismi antichi, delineando altresì prospettive comparative e analitiche. combination of attributes capable of identifying them with more or less certainty, what the ancient authors called insignia, the "special signs" that guided the interpretation / identification of a signum. In particular, some concrete objects will be examined, used by the Roman culture to construct divine images in their function of attributes endowed with a specific identity. In Rome there were also specific cases in which the power of the insigne was overflowing. The mark of recognizability, in these cases, was identified with the totality of the image, just as the image was reduced to the extension of the mark of recognition: the insigne, in that case, was the image. This will lead us to question the methods of construction, adaptation, borrowing and exclusion of divine images among ancient polytheisms, outlining comparative and analytical perspectives.

\section{PAROLE CHIAVE}

Appropriazione; identità; immagini divine; insignia; signa panthea; statua

\section{KeYWORDS}

Appropriation; divine images; identity; insignia; signa panthea; statue 
Il Dibattito RELATIVO ALla "CORPOREitÀ" e alla trasposizione in immagini del divino è già antico ed è stato oggetto di numerose inchieste da parte degli studiosi moderni. A partire dal bilancio offerto da Corps des dieux di Charles Malamoud e Jean Pierre Vernant, ${ }^{1}$ dagli anni 2000 è la questione della figurazione del corpo delle divinità antiche ad essere stata maggiormente affrontata in monografie ed opere collettive: basti ricordare i tre volumi pubblicati a Rennes tra 2006 e 2008 nei Cahiers $d^{\prime}$ 'histoire $d u$ corps antique e le riflessioni in rapporto all' antropologia dell' immagine e alla storia dell' arte; ${ }^{3}$ infine il ruolo fondamentale ricoperto dagli specialisti dei politeismi antichi secondo la duplice prospettiva di analisi della costruzione visuale e dell'efficacia cultuale delle rappresentazioni figurate del divino nel mondo antico. ${ }^{4}$ Tali modi di rappresentazione erano molteplici, inestricabilmente interconnessi l'uno all'altro: plastici (la figurazione degli dèi), politici (il ruolo attribuito alle potenze superiori nella condotta quotidiana della collettività), simbolici (nei rituali), concettuali (all' interno dei sistemi teologici), narrativi (i racconti mitici). La questione di questa doppia dinamica della "fabbrica del divino" (così come viene definita da Belayche e Pirenne-Delforge) concerne dunque da una parte la nostra comprensione di ciascuna divinità in particolare e dall' altra la nostra capacità di rilevare le strutture e i funzionamenti di insiemi divini complessi rimodellabili e diversamente costruiti. ${ }^{5}$

1. Malamoud e Vernant, 1986.

2. Bodiou, Frère e Mehl, 2006; Prost e Wilgaux, 2006; Dasen e Wilgaux, 2008; Garelli e Visa-Ondarçuhu, 2008.

3. Gordon, 1979; Elsner, 2007; Belting, 1998 e 2004.

4. Gladigow, 1985-1986; 1990; 1994; Dewailly, 2001; Estienne e Lissarague, 2005; Estienne, Jaillard, Lubtchansky e Pouzadoux, 2008; Mylonopoulos, 2010; Borgeaud e Fabiano, 2013; Estienne, Huet, Lissarrague e Prost, 2015; Pirenne-Delforge e Belayche, 2015.

5. Pirenne-Delforge e Belayche, 2015, p. 5. 
Obiettivo di questo lavoro è osservare limitatamente al mondo romano il problema posto dal corpo divino nella sua trasposizione in immagini; in altre parole, indagare in che modo le divinità antiche vennero pensate e prodotte in immagini culturali realizzate per descrivere e organizzare le molte divinità che popolavano il loro pantheon. Ci si concentrerà, in primo luogo, sulle modalità attraverso le quali gli dèi vennero dotati di un corpo attraverso degli artefatti, e successivamente si indagherà il rapporto che la cultura romana intratteneva con essi. Si passerà infine ad analizzare come tali divinità venissero conosciute attraverso le loro effigi e quindi come si rendessero riconoscibili all'osservatore, attraverso singoli attributi che ne permettevano l'identificazione.

\section{LA “PERSONALIZZAZIONE” DEL DIVINO COME STRATEGIA PERCETTIVA IN AMBITO RELIGIOSO: “VEDERE” GLI DÈI A ROMA}

Interrogandosi sulle rappresentazioni degli dèi, Erodoto ${ }^{6}$ attribuiva un ruolo fondamentale nella sistematizzazione della teogonia ellenica ad Omero ed Esiodo: i due poeti avrebbero elaborato nelle loro opere il profilo generale di ciascuna divinità, indicandone denominazioni, genealogie, prerogative, attributi e precisandone inoltre l'aspetto esteriore. ${ }^{7}$ Anche a Roma, secondo una concezione filosofica diffusa, in origine non esisteva nessun simulacro degli dèi, né dipinto, né scolpito, e non esistevano neppure templi e cappelle, ma solo semplici altari; fu Numa a vietare di erigere immagini divine traendo spunto da animali o da uomini. ${ }^{8}$ Varrone ad esempio, nelle Antiquitates rerum divinarum, affermava che la più antica e nobile usanza fosse quella di venerare gli dèi senza ricorrere a nessuna immagine; in seguito essi avrebbero trovato una forma, ognuno la propria, affinché potessero essere riconosciuti e vene-

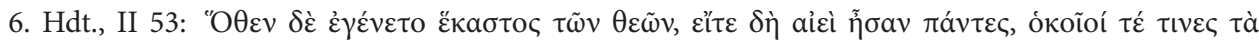

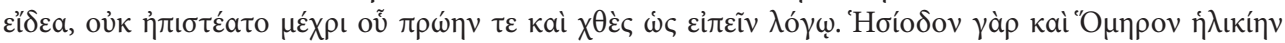

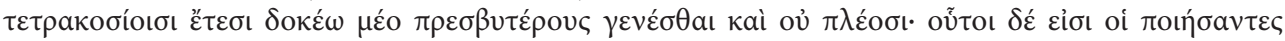

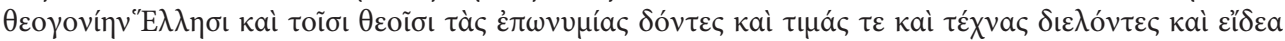

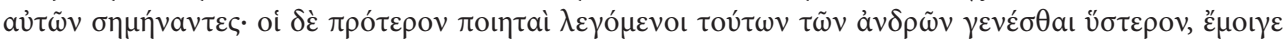

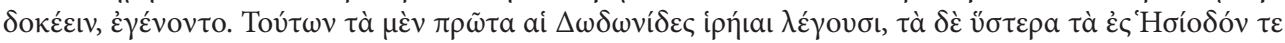

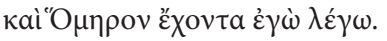

7. Pironti, 2012, p. 42.

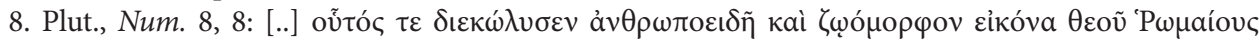

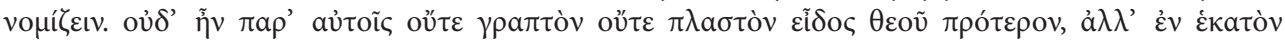

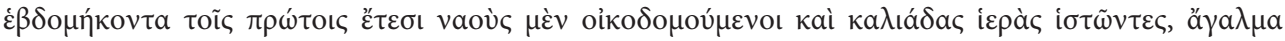

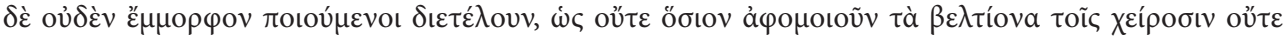

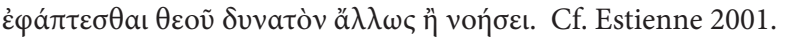


rati sotto sembianze umane. ${ }^{9}$ Si pervenne ad commenticios et fictos deos, cioè a dèi immaginati e plasmati secondo la fantasia degli uomini; così furono noti l'aspetto, l'età, l'abbigliamento, gli attributi, la genealogia, i matrimoni e la parentela e tutto fu trasformato a somiglianza della fragilità umana:

"Queste credenze furono ulteriormente promosse dai poeti, pittori, artigiani, perché non era facile rappresentare sotto la sembianza di altre forme gli dèi in azione e in attività (agentes et molientes)". ${ }^{10}$

Il corpo si configurava innanzitutto come medium per pensare il divino, ma permetteva altresì di oggettivare una presenza divina secondo modalità estremamente variegate. Ma gli dèi sono degli esseri polimorfi, estremamente variegati nel loro "tissu identitaire". ${ }^{11}$ Se essi hanno un corpo, essi non sono limitati ad un solo corpo, a una sola rappresentazione convenzionale o congetturale. ${ }^{12}$ A partire da questo, la questione che si pone è quella del rapporto tra l' immagine cultuale (la statua) con il corpo (reale e polimorfico) degli dèi. Può il corpo di un dio essere identificato con la sua statua, la sua effigie? In effetti, gli dèi si manifestavano costantemente agli uomini, i quali avevano così la possibilità di entrare in contatto con essi, attraverso una gamma di sensazioni (colori, suoni, odori etc.) ${ }^{13}$ tra le quali quella della vista giocava un ruolo chiave; essa era percepita come la facoltà sensoriale primaria ed ogni «oggetto» conoscibile doveva ricadere sotto tale facoltà in modo da essere percepibile:

"Simonide, o chi ne fu l' inventore, ha visto bene: nella nostra mente prendono forma soprattutto le immagini delle cose trasmesse ed impresse dai sensi; fra tutti i nostri sensi, il più acuto è la vista: perciò è possibile ricordare con molta facilità ciò che abbiamo percepito con l' udito o il pensiero se lo consegniamo alla mente anche con l'aiuto della vista; così una forma visibile o un' immagine o un'effigie possono indicare cose nascoste

9. August., De Civ. D. IV 31: Antiquos Romanos plus annos centum et sptuaginta deos sine simulacro coluisse. Quod si adhuc [..] mansisset, castius dii observarentur [..] qui primi simulacra deorum populis posuerunt, eos civitatibus suis et metum dempsisse et errorem addidisse. Cf. Estienne, 2001, p. 190.

10. Cic., Nat. D. I 77: Auxerunt autem haec eadem poetae, pictores, opifices, erat enim non facile agentes aliquid et molientes deos in aliarum formarum imitatione servare (trad. C.M. Calcante 1996).

11. Bettini in Pironti e Bonnet, 2017, p. 41: "c'est précisément dans la multiplicité et variété du tissu identitaire, dans la capacité d'être, de manière sérielle, plusieurs, et non pas un seul- soi même et autre que soi-même- que réside le privilège des dieux".

12. Malamoud e Vernant, 1986, pp. 1-10.

13. Cf. Grand-Clément in Pironti e Bonnet, 2017, pp. 43-61. 
e che non cadono sotto il giudizio della vista con tanta efficacia da renderci quasi visibile ciò che a stento possiamo abbracciare con il pensiero". ${ }^{14}$

Nonostante gli dèi fossero dotati di un "sangue-non sangue" e di un "corponon corpo", ${ }^{15}$ era proprio attraverso quelle "rappresentazioni" così antropomorfe realizzate da poeti e artisti che si potevano intellegere gli dèi. ${ }^{16}$ La statua di una divinità si imponeva come un polo attorno al quale si organizzava il funzionamento del santuario; ${ }^{17}$ molti documenti iconografici o letterari, infatti, mostrano che la statua del dio poteva essere al centro dell' attenzione fino ad un attaccamento morboso e quasi ridicolo, ${ }^{18}$ come polemizza Agostino che sembra far risalire questo rimprovero a Seneca stesso:

"Ma va' in Campidoglio, ti farà vergognare della frenesia esposta al pubblico ciò che una
stravagante mania si è attribuita come dovere. Un tale fa vedere alcuni nomi a un dio,
un altro notifica le ore a Giove, qualcuno fa il gesto del littore, un altro unge, giacché un
inutile movimento delle braccia imita chi spalma l' unguento. Vi sono delle donne che
pettinano i capelli a Giunone e a Minerva; in piedi lontano dal tempio e non soltanto
dalla statua muovono le dita col gesto delle acconciatrici. Altre sostengono lo specchio.
Vi sono alcuni che invitano gli dèi ad andare con loro per ottenere la cauzione, altri
fanno vedere loro lo scritto di ricorso e fanno loro conoscere il processo che li riguarda.
Un colto primo attore, ormai vecchio decrepito, eseguiva ogni giorno in Campidoglio

14. Cic., De Or. II 357: Vidit enim hoc prudenter sive Simonides sive alius quis invenit, ea maxime animis effingi nostris, quae essent a sensu tradita atque impressa; acerrimum autem ex omnibus nostris sensibus esse sensum videndi; qua re facillime animo teneri posse ea, quae perciperentur auribus aut cogitatione, si etiam commendatione oculorum animis traderentur; ut res caecas et ab aspectus iudicio remotas conformatio quaedam et imago et figura ita notaret, ut ea, quae cogitando complecti vix possemus, intuendo quasi teneremus (trad. R. Sabbadini 1970).

15. Cic., De Nat. D. I 77: Idem facit in natura deorum: dum individuorum corporum concretionem fugit, ne interitus et dissipatio consequatur, negat esse corpus deorum, sed tamquam corpus, nec sanguinem, sed tamquam sanguinem. Mirabile videtur quod non rideat haruspex, cum haruspicem viderit; hoc mirabilius, quam vos inter vos risum tenere possitis? "Non est corpus, sed quasi corpus": hoc intellegerem, quale esset, si in cereis fingeretur aut fictilibus figuris; in deo quid sit quasi corpus aut quid sit quasi sanguis, intellegere non possum. Ne tu quidem Vellei, sed non vis fateri.

16. Lo stesso procedimento è ben mostrato anche per il versante greco da Mylonopoulos, 2010, p. 179: "[..] attributes were indeed powerful instruments of communication, because they transported significant, cognitive information by means of visual, non-verbal signs. In antiquity, attributes were certainly of great help to viewers trying to understand the divine in its micro-individuality manifested in a specific, very concrete image".

17. Rüpke, 2010, pp. 182-183.

18. Estienne, 2001, p. 192. 
una sua rappresentazione, convinto che gli dèi lo seguissero di buon grado, perché gli uomini avevano cessato di farlo. Ogni categoria di artigiani se ne sta lì con le mani in cintola a lavorare per gli dèi immortali”. ${ }^{19}$

Anche per Varrone (secondo un frammento riportato nel De civitate dei di Agostino $)^{20}$ l' aspetto che le divinità rivelavano (simulacra), insieme agli attributi (ornatus, insignia) che li connotavano, permettevano di accedere ad una migliore comprensione del divino, stabilendo una relazione fondata sulla fiducia nella loro bontà e non sulla paura. È proprio all' interno di questo celebre passo che si tocca il cuore della terminologia utilizzata nella cultura romana per designare le effigi divine. Come in greco, ${ }^{21}$ esisteva in latino una pluralità di sostantivi attribuibili alla figurazione cultuale, anche se bisogna guardarsi dalla convinzione che nelle società sia greca che romana esistesse una netta distinzione tra "immagine di culto" e "immagine votiva", tra "immagine del dio" e "immagine offerta al dio". ${ }^{22}$ Infatti, pur possedendo la nozione di "rappresentazione figurata", non esisteva alcuna parola ben definita per designare la "statua di culto" così come noi oggi la concepiamo: ${ }^{23}$ ad esempio, la moltitudine dei termini usati in Grecia a proposito delle rappresentazioni divine, ognuno dei quali era connotato in base al contesto in cui si manifestava (agalma, xoanon, hedos, bretas, andrias, eikon, hydrima), rivelava una comprensione fluida delle funzioni e delle

19. August., De Civ. D. VI 10, 2: In Capitolium perveni, pudebit publicatae dementiae, quod sibi vanus furor attribuit officii. Alius nomina Deo subicit, alius horas Iovi nuntiat: alius lictor est, alius unctor, qui vano motu bracchiorum imitatur unguentem. Sunt quae Iunoni ac Minervae capillos disponant (longe a templo, non tantum a simulacro stantes digitos movent ornantium modo), Sunt quae speculum teneant; sunt qui ad vadimonia sua deos advocent, sunt qui libellos offerant et illos causam suam doceant. Doctus archimimus, senex iam decrepitus, cotidie in Capitolio mimum agebat, quasi dii libenter spectarent, quem illi homines desierant. Omne illic artificum genus operatum diis immortalibus desidet (trad. L. Alici 2001).

20. August., De Civ. D. VII 5 = Varro, Antiquitates divinarum, fr. 225: [..] sic Varro commendat ut dicat antiquos simulacra deorum et insignia ornatusque finxisse, quae cum oculis animadvertissent hi qui adissent doctrinae mysteria possent animam mundi ac partes eius, id est deos veros, animo videre; quorum qui simulacra specie hominis fecerunt, hoc videri secutos quod mortalium animus, qui est in corpore humano, simillimus est inmortalis animi.

21. Vernant, 1979 e 1990.

22. Estienne in Mylonopoulos, 2010, p. 257: "As a matter of fact, the gap between the modern notion of 'cult statue' and the literary and epigraphic evidence does not allow the identification of an unambiguous and coherent category of divine representations".

23. Linant de Bellefonds, 2004, introduction: "Il est d' usage, dans le vocabulaire moderne, de distinguer les images 'votives', simples offrandes à la divinité, de la statue 'cultuelle', seule véritable représentation du dieu vivant. Or, il faut bien reconnaître que les sources écrites, pas plus que l' iconographie ou l'archéologie, ne nous apportent de certitudes à ce sujet. Il n'existe, ni in grec ni en latin, de terme précis désignant explicitement une statue de culte". 
forme di un'effigie usata nel rituale. ${ }^{24} \mathrm{Il}$ vocabolario romano invece, apparentemente meno ricco, era sicuramente un po' più chiaro rispetto al dispersivismo lessicale greco; termini come effigies, imago, signum, simulacrum, statua erano tutti sostantivi che designavano degli artefatti, dei prodotti umani. ${ }^{25}$ Signum era il termine più frequentemente usato in questo contesto e vari erano i significati legati a questa parola; la sua etimologia è oscura anche vi è comune accordo nel significato originario concreto di "marchio, traccia, impronta". Questa è la definizione che ne dà Cicerone: signum est quod sub sensum aliquem cadit et quiddam significat, ${ }^{26}$ cioè un oggetto la cui presenza sensibile indica un' altra realtà o verità, che il signum evoca in un modo o in un altro; attraverso tale "segnale" tali realtà o verità vengono rese intellegibili all' uomo. Spostandoci nel campo della statuaria, signum, la "statua", era la rappresentazione di un dio, il "segno" che era stato messo al suo posto per indicarne la presenza all' interno del santuario. Se accettiamo questa etimologia bisogna ammettere che signum non avesse come primo significato la statua come artefatto concreto, confezionato con questo o quel materiale, ma quello di segno del dio, di simbolo che lo rappresentava. D'altra parte il termine signum designante la statua era spesso seguito in latino dal genitivo del nome del dio, e ciò marcava l'appartenenza del signum al dio e non la completa identificazione tra i due. ${ }^{27}$ Si può altresì aggiungere che signum era raramente impiegato senza determinante e quando ciò avveniva si trattava di casi in cui il contesto non permetteva alcuna ambiguità. Ciò ci porta a supporre che la cultura romana avesse ben chiara e delineasse nettamente la differenza tra il dio in persona e la sua rappresentazione e non pensasse affatto di "vederlo" in tutta la sua essenza quando aveva di fronte la sua statua. Il secondo termine impiegato per designare le statue divine era simulacrum. L'etimologia stessa della parola, che rinvia chiaramente all' aggettivo similis, "somigliante", indicava l'affinità con il registro dell' imitazione e non più, come nel caso di signum, con il registro del simbolo. Tale termine non si applicava soltanto alle statue degli dèi, ma designava tutte le forme di copia, di riproduzione di un'apparenza e quindi, implicitamente, di illusioni ingannatrici. Simulacrum, inoltre, come signum, era spesso seguito dal nome del dio e ciò esprimeva un'appartenenza che situava la statua in un rapporto particolare con il dio stesso:

24. Benveniste, 1932, pp. 118-122; Bettinetti, 2001, pp. 25-63; Mylonopoulos, 2010, p. 5.

25. Dubourdieu in Borgeaud e Fabiano, 2013, p. 28.

26. Cic., Inv. rhet. I 48.

27. Dubourdieu, in Borgeaud e Fabiano, 2013, p. 29; Brachet 1994, p. 35. Signum Herculis: CIL I²626; Plaut., Rud. 823; CIL VI 278; 597; 607; signum Veneris: Plaut., Rud. 560, 648, 823; CIL VI 778; signum Iovis: CIL VI 36787; Cic., Div. I 77; II 47; signum Martis: CIL VI 479; Cic., Fam. VII 23, 2: Liv., Hist. XXII 1, 12; signum Cereris: Cic., Verr. 4, 74; 4, 110; signum Volcani: CIL I²2247; signum Liberi: CIL VI 460. 
"l'icona", infatti, gli apparteneva nella misura in cui essa era la sua copia, ma essa non era lui, ne era semplicemente la riproduzione. In definitiva, perciò, le differenti modalità secondo le quali la divinità si rendeva visibile agli uomini avevano in comune la duplice lettura di presenza-assenza della divinità: le statue divine erano, in primo luogo e a titoli diversi, delle immagini degli dèi forgiate dagli uomini e per gli uomini affinché si potessero percepire attraverso la vista, non le divinità stesse; tuttavia si poteva pensare anche ad una manifestazione attiva e sensibile degli dèi fra loro attraverso le loro statue. ${ }^{28}$ Anche nei confronti delle effigi divine, perciò, vi erano due nature simultanee, due vie, anch'esse non contraddittorie, attraverso le quali si potevano pensare gli dèi, una volta visibili e sensibili fra gli uomini, ma allo stesso tempo irrimediabilmente assenti.

A questo punto sarà necessario soffermarsi a riflettere su come tali divinità venissero conosciute attraverso le loro effigi e quindi come si rendessero riconoscibili: infatti, dal momento che la città era piena di dèi e in ogni angolo sorgevano rappresentazioni divine, si rivelava di fondamentale importanza, per ogni cittadino, conoscere chi si aveva di fronte. Quali erano, dunque, i signa utilizzati per fabbricare le figurazioni del divino in modo che esse fossero identificabili come questo o quel personaggio della schiera delle potenze divine? Prima di rispondere a questa domanda, tuttavia, sarà altrettanto importante e chiarificante analizzare quale fosse la configurazione culturale che venne impiegata, a livello emico, per parlare di "identità personale" in generale; in seguito a questo, si vedrà come la cultura romana si servì di tale concetto relativamente alle proprie divinità.

\section{L'IDENTITÀ PERSONALE A ROMA COME ESTERIORITÀ RICONOSCIBILE E LA COGNITIO DEGLI DÈI}

Se si cerca, innanzitutto, dove la cultura romana collocava l' identità personale, si nota come gli autori antichi insistessero su termini legati all'apparenza esteriore; come nota Bettini, essi venivano legati a nozioni come cognitio o notitia, espressioni che ricollegabili al verbo gnosco "conoscere" prendevano in considerazione, perciò, la "nostra" identità individuale in termini di "riconoscibilità." ${ }^{29}$ In latino cognitio indicava specificamente la riconoscibilità / identificazione di qualcuno o di qualcosa, ovvero ciò che in greco era espresso con il termine ảva $\gamma v \omega$ ó

28. Si veda a tal proposito Ando, 2010.

29. Bettini, 2000, pp. 314-315; Isid., Etym. XI 13: facies [..] ibi est [..] tota figura hominis et unius cuisque personae cognitio; Diff. II 52: facies dicta est, eo quod notitiam faciat hominis. 
al termine cognitio, si tratta di identificare qualcuno o qualcosa, appunto, "riconoscendolo". ${ }^{30}$ Così infatti Isidoro di Siviglia, nelle sue Differentiae, tracciava la linea di confine tra cognitio e agnitio: se l' agnitio è ciò che già si conosce e che si può ricordare in seguito, la cognitio risulta essere ciò di cui non si aveva conoscenza e che appunto si impara a conoscere successivamente. ${ }^{31}$ A questo punto si pone spontanea una domanda: la cultura romana ha impiegato il termine cognitio e dunque tutta la famiglia del verbo nosco a proposito dell' identità delle immagini divine, ovvero nel momento in cui gli dèi si esplicavano in forma visuale? Già Agostino nel De civitate dei criticava l'affermazione di Varrone per cui una corretta cognitio della divinità fosse fondamentale per comprenderne la vis, la facultas e la potestas, nonché per un'efficace invocazione:

\begin{abstract}
"Per qual ragione dunque Varrone si vanta di rendere un grande servizio ai suoi concittadini perché non solo ricorda gli dèi che si devono adorare dai Romani, ma espone anche la mansione di ciascuno? Non giova nulla, egli dice, conoscere il nome e la figura di un medico e ignorare che è medico; così, soggiunge, non giova nulla sapere che Esculapio è un dio, se non sai che protegge la salute e perciò non sai il motivo per cui lo devi invocare. Lo conferma anche con un'altra similitudine. Dice che non solo non si può vivere agiatamente ma che non si può vivere affatto se non si conoscono il falegname, il mugnaio e il muratore, cui poter chiedere un servizio, ovvero se non si sa chi assumere come collaboratore, guida e insegnante. Allo stesso modo, egli afferma, non v'è dubbio che è utile la conoscenza degli dèi (cognitionem deorum), se si sa anche quale forza ( $v i s$ ), facoltà (facultas) e potere (potestas) ha ciascun dio sulle varie cose. Da questo potremo conoscere, egli dice, quale dio, secondo la competenza di ciascuno, dobbiamo chiamare in aiuto e invocare per non comportarci come i mimi e non chiedere l'acqua a Bacco e il vino alle Linfe. È davvero una grande utilità. Ma chi non ringrazierebbe Varrone se affermasse il vero e insegnasse agli uomini ad adorare l'unico vero Dio da cui deriva ogni bene?".32
\end{abstract}

30. TLL, s.v. cognitio, p. 1483: [..] actio recognoscendi quem anagnorismòn appellant (spectat ad homines).

31. Isid., Diff. I 89.

32. August., De Civ. D. IV 22: Quid est ergo, quod pro ingenti beneficio Varro iactat praestare se civibus suis, quia non solum commemorat deos, quos coli oporteat a Romanis, verum etiam dicit quid ad quemque pertineat? Quoniam nihil prodest, inquit, hominis alicuius medici nomen formamque nosse, et quod sit medicus ignorare: ita dicit nihil prodesse scire deum esse Aesculapium, si nescias eum valetudini opitulari atque ita ignores cur ei debeas supplicare. Hoc etiam alia similitudine affirmat dicens, non modo bene vivere, sed vivere omnino neminem posse, si ignoret quisnam sit faber, quis pistor, quis tector, a quo quid utensile petere possit, quem adiutorem assumere, quem ducem, quem doctorem; eo modo nulli dubium esse asserens ita esse utilem cognitionem deorum, si sciatur quam quisque Deus vim et facultatem ac potesta- 
Anche un'elegia dei Priapea ci fornisce alcune informazioni utili e stabilisce un legame tra la famiglia linguistica di nosco (o di cognitio) e quella che noi potremmo definire l'identità delle immagini:

"Ciascuno fra noi ha forme del corpo che sono riconosciute (notas habemus quisque corporis formas): Febo è capelluto, Ercule membruto, il tenero Bacco ha un aspetto verginale. Esculapio ha sempre una lunga barba, nessuno è più pettoruto del feroce Marte, ma non vi è dio più dotato sessualmente di Priapo".33

Ciascun dio, perciò, ovvero l'immagine di ciascun dio, era notus, cioè riconoscibile proprio come tale e dotato di una propria cognitio. Generalmente, infatti, la rappresentazione iconografica di ciascuno di essi possedeva un qualche attributo o combinazione di attributi capaci di identificarlo sia pure con maggiore o minore certezza. La connessione tra cognitio e facies come termini per l' identificazione visuale degli dèi è espressa anche a chiare lettere dall'accademico Cotta nel De natura deorum; il filosofo, criticando l'epicureio Velleio che affermava che gli dèi non potessero non avere aspetto umano (e dunque dotava gli dèi degli stessi difetti umani), arrivava alla conclusione (se essi, al contrario, non hanno alcun difetto perché sono perfetti) che esistesse una sola forma per tutte le divinità:

"Pensiamo che essi, pur non essendo così gravemente strabici, lo siano un poco, o che abbiano nei, il naso camuso, le orecchie pendenti, una fronte e una testa sproporzionate? Sono forse privi di tutti questi difetti che gli uomini possiedono? Ve lo concedo; forse hanno tutti lo stesso viso? Se ne hanno parecchi, un viso deve essere necessariamente più bello di un altro, dunque un dio può essere privo della bellezza suprema; se hanno tutti lo stesso viso, l'Accademia deve avere un gran successo in cielo perché, se non vi è nessuna differenza tra gli dèi, non ci sono né conoscenza (cognitio), né percezione (perceptio) fra gli dèi”. ${ }^{34}$

tem cuiusque rei habeat. Ex eo enim "poterimus, inquit, scire quem cuiusque causa deum advocare atque invocare debeamus, ne faciamus, ut mimi solent, et optemus a Libero aquam, a Lymphis vinum". Magna sane utilitas. Quis non huic gratias ageret, si vera monstraret, et si unum verum Deum, a quo essent omnia bona, hominibus colendum doceret? (trad. L. Alici 2001).

33. Priapea, XXXVI: Notas habemus quisque corporis formas: / Phoebus comosus, Hercules lacertosus, / trahit figuram virginis tener Bacchus... intonsa semper Aesculapio barba est, / nemo est feroci pectorosior Marte: / quod si quis inter haec locus mihi restat, / deus Priapo mentulatior non est (trad. personale). Cf. Bettini, 2016, p. 168.

34. Cic., De Nat. D. I 80: Redeo ad deos. Ecquos si non tam strabones at paetulos esse arbitramur, ecquos naevum habere, ecquos silos, flaccos, frontones, capitones, quae sunt in nobis, an omnia emendata in illis? Detur id vobis; num etiam una est omnium facies? Nam si plures, aliam esse alia pulchriorem necesse est, 
Se gli dèi dunque avessero una sola facies non sarebbe possibile "percepirli" (perceptio), né "riconoscerli" (cognitio) in quanto tali; ma essi, come gli uomini, non sono tutti uguali, anzi, è proprio grazie ai pittori e agli scultori che essi hanno ricevuto una forma (ea facie, ornatu, aetate, vestitu) ben determinata che li distingue gli uni dagli altri e che, come tale, viene conosciuta (noscere):

"Fin da piccoli infatti conosciamo Giove, Giunone, Minerva, Nettuno, Vulcano, Apollo e gli altri dèi con quell'aspetto che hanno voluto pittori e scultori; e non solo con quell'aspetto, ma con quell'equipaggiamento, con quell'età, con quel vestito". ${ }^{35}$

Attraverso l'ars, perciò, gli uomini arrivano a "conoscere" gli dèi e a dotarli di un' identità. Ma su cosa poteva riporre la cognitio degli dèi? Ovvero, quali erano in concreto gli oggetti impiegati dalla cultura romana per costruire le rappresentazioni figurate degli dèi dotate di una propria "identità"?

\section{LA RICONOSCIBILITÀ DEGLI DÈI ATTRAVERSO GLI INSIGNIA}

In questa prospettiva, sembra che la "riconoscibilità" si realizzasse soprattutto attraverso ciò che i Romani designavano con il termine insignia o, con termini meno pregnanti, nota, argumentum, ornatus e habitus. Dal punto di vista etimologico, l'aggettivo insignis era formato da signum ( la manifestazione "sensibile" ed "efficace" della divinità) e il preverbio, di valore intensivo, in-. Varie definizioni sono state date di questo aggettivo: nel Lewis Short "distinguished by a mark; remarkable; noted, eminent, distinguished prominent, extraordinary", ${ }^{36}$ nell' Ernout-Meillet "distingué par une marque particulière", ${ }^{37}$ nell' Oxford Latin Dictionary "clearly visible or recognizable, easily apprehensible, remarkable, having a special significance, ${ }^{38}$ anche se la definizione più pertinente, come sottolinea Bettini, sembra quella offerta dal Lexicon Totius Latinitatis: insignis est qui signo aliquo vel nota inter alia eminet: in quo est aliquod

igitur aliquis non pulcherrimus deus; si una omnium facies est, florere in caelo Academiam necesse est: si enim nihil inter deum et deum differt, nulla est apud deos cognitio, nulla perceptio (trad. C. M. Calcante 1996). Cf. Bettini, 2016, p. 169.

35. Cic., De Nat. D. I 81: [..] a parvis enim Iovem, Iunonem, Minervam, Neptunum, Vulcanum, Apollinem, reliquos deos ea facie novimus, qua pictores fictoresque voluerunt, neque solum facie, sed etiam ornatu, aetate, vestitu (trad. C. M. Calcante 1996).

36. Lewis-Short, s.v. insignis, p. 965.

37. DELL, s.v. signum, p. 624.

38. OLD, s.v. insigne-is, p. 924. 


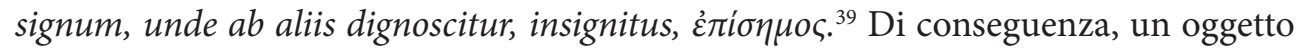
poteva essere definito insignis quando portava su di sé un signum che "spiccava", che si distingueva: un signum, dunque, che nel suo essere "segnale", faceva risaltare l'oggetto in questione fra gli altri. ${ }^{40}$ Tale aggettivo, infatti, poteva riferirsi generalmente al corpo degli animali per farne risaltare qualche caratteristica particolare rispetto al gruppo di appartenenza (de notis a natura in corpore impressis) ${ }^{41}$ oppure a quello degli uomini per indicarne delle marche distintive, sia in senso positivo che negativo ${ }^{42}$ (soprattutto caratteriali: universim dicitur de eo qui ob decus aliquod facile incurrit in oculos et aliis distinguitur, singularis, praestans, eximimus, egregius). ${ }^{43}$ Ciò che veniva connotato con questo aggettivo, perciò, diventava immediatamente manifesto a tutti, chiaramente visibile e riconoscibile per le sue caratteristiche e per questo distinguibile e degno di nota. ${ }^{44}$ Esso poteva riferirsi sia agli uomini che agli dèi denotandone una marca di riconoscimento e di identificazione rispetto agli altri: Pelope era insignis per l'omero eburneo (umeroque Pelops insignis eburno), ${ }^{45}$ Apollo per la folta chioma (crinibus insignis Phoebus) ${ }^{46}$ e Bacco per le sue corna (insignis cornu Bacchus). ${ }^{47} \mathrm{Se}$

39. Forcellini, 1965, s.v. insignis-e, p. 867.

40. Bettini, 2016, p. 173.

41. Forcellini, 1965, s.v. insignis-e, p. 867. Fest., De verb. sign. p.114: Insigne appellantur boves, qui in femine et in pede album habent, quasi insigniti; Verg., G. III 56-59: nec mihi displaceat maculis insignis et albo / aut iuga detractans interdumque aspera cornu / et faciem tauro proprior quaeque ardua tota / et gradiens ima verrit vestigia cauda; Plin., HN VIII 15, 38: Ceterorum animalium, quae modo convecta undique Italiae contigere saepius, formas nihil attinet scrupulose referre. paucissima Scythia gignit inopia cruticum, pauca contermina illi Germania, insignia tamen boum ferorum genera, iubatos bisontes excellentique et vi et velocitate uros, quibus inperitum volgus bubalorum nomen inponit, cum id gignat Africa vituli potius cervique quadam similitudine.

42. Forcellini, 1965, s.v. insignis-e, p. 867. Cic., Leg. III 8, 19: Insignis ad deformitatem puer; Suet., Calig. 26: Insignis debilitate aliqua corporis.

43. Forcellini, 1965, s.v. insignis-e, p. 867. Verg., Aen. VI 404: Troius Aeneas, pietate insignis et armis; Luc., Phar. VIII 73: insignis titulis avorum; Cic., Amic. 27, 102: Virtus Scipionis etiam posteris erit clara et insignis.

44. (facilmente riconoscibile) Liv., X 15, 5: nec valli tantum ac fossarum vestigia relicta sed multo illis insigniora monumenta vastitatis circa regionumque depopulatarum; Hor., Carm. I 33, 5: [..] insignem tenui fronte Lycorida; (facilmente apprendibile) Cic., Clu. 61: iudicia senatoria...vera atque insigni turpitudine notata; Liv., XLII 6, 2: insigne adversus Persea odium Romanorum fecit. OLD, s.v. insigne-is, p. 924: "Something worn or carried as an indication of rank, status, identity, etc.; a personal decoration or other concrete mark of honour".

45. Verg., G. III 6-8: quoi non dictus Hylas puer et Latonis Delos / Hippodamemque umeroque Pelops insignis eburno / acer equis.

46. Ov., Am. I 1, 11-12: Crinibus insignem quis acuta cuspide Phoebum / instruat, Aoniam Marte movente lyram?

47. Ov., Ars Am. III 347-348: O ita, Phoebe, velis, ita vos pia numina vatum / insignis cornu Bacche novemque deae. 
dal singolare ci spostiamo ai suoi usi plurali molto più frequenti, il termine insignia, come "note" o segni di riconoscibilità, era presente nell' identificazione degli eserciti tramite vessilli o signa militari, ${ }^{48}$ nella designazione di premi corrispondenti a vittorie riportate sul campo di battaglia o in altri tipi di certamina, ${ }^{49} \mathrm{e}$ anche nella definizione degli honores magistratum (fossero essi effettivamente dei magistrati dello stato, consules o imperatores ${ }^{50} \mathrm{o}$ dei sacerdoti $\left.{ }^{51}\right)$, indicando appunto ciò che li "contrassegnava", ovvero ciò che li identificava come persone investite di una funzione pubblica. Anche nel campo delle immagini divine, gli insignia funzionavano alla stregua di notae, ovvero come dei segni di riconoscibilità e identità. ${ }^{52}$ Tuttavia se la statua a Roma (ovvero l' immagine del dio) si definiva genericamente signum, ciò che in definitiva ne rendeva possibile la cognitio e dunque la "conoscibilità" e la "riconoscibilità" fra tutti gli altri manufatti non potevano che essere gli insignia, quei signa, appunto, che "risaltavano" (in-) tra l' insieme degli elementi che ne formavano complessivamente l' immagine connotandosi come un "signum più che signum". ${ }^{53}$ Tali oggetti creavano una linea continuativa tra gli uomini e gli dèi che, attraverso tali insignia, esplicavano la propria "identità" in termini visuali: Castore e Polluce, ad esempio, si riconoscevano immediatamente per via delle stelle d'oro che costituivano i loro insignia ([...] eorum insignia deorum, stellae aureae, quas dixi, Delphis positae), ${ }^{54}$ allo stesso modo in cui la Mater Magna aveva sul capo, come insigne, una corona murale (muralique caput summum cinxere corona, / eximiis munita locis quia sustinet urbes. /quo nunc

48. TLL, s.v. insignis, pp. 1898-1901. Varro, Ling. VII 37: haec insignia atque ornamenta militaria; Tac., Hist. I 82, 3: insignia militiae; I 38, 3: ut praetorianus ut legionarius insignibus suis distingueretur; Liv., Hist. IX 40, 1: ut acies... fulgeret novis armorum insignibus; Caes., BGall. I 22, 2: [..] id se a Gallicis armis atque insignibus cognovisse.

49. Stat., Theb. VI 643: finiti cursus operumque insignia praesto; Firm., Math., VI, 31, 3: athleta victor famosa reportet insignia coronarum; Caes., BCiv. III 71, 3: neque in fascibus insignia laureae praetulit; Verg, Aen. VIII 683: cui, belli insigne superbum, tempora navali fulgent rostrata corona; Liv., VII 37, 3: his decoratus insignibus (sc. coronis).

50. Cic., Clu. 154: toga praetexta, sella curulis, insignia, fasces; Sall., Cat. 51, 38: insignia magistratum; Liv., Hist. II 1, 8: Omnia rura, omnia insignia primi consules tenuere; Tac., Hist. IV 4, 2: imperio et insignibus consularibus; I 8: quae ita sancta generi hominum agresti fore ratus, si se ipse [Romulus] venerabilem insignibus imperii fecisset.

51. CIL I 10: quei apice insigne dialis flaminis gesistei; Verg., Aen. X 539: sacerdos [..] totus conlucens veste atque insignibus albis; Liv., X, 7, 9: pontificalia atque auguralia insignia.

52. Varro, Sat. Men. 170: insignibus Martis; Val. Fl., Arg. VI 598: insignia Bacchi induit; IX 776: te, dea (sc. Isis) .. tuaque haec insignia vidi; Plin., HN XXXVII, 5: [..] ut Musis quoque singulis sua redderentur insignia; Stat., Theb. X 254: [..] Phoebea insignia, frondes.

53. Bettini, 2016, p. 174.

54. Cic., Div. I 75. 
insigni per magnas praedita terras / horrifice fertur divinae matris imago $)^{55} \mathrm{e}$ Giove i suoi specifici attributi (det pignus amoris, / si modo verus is est; quantusque et qualis ab alta / Iunone excipitur, tantus talisque, rogato, / det tibi conplexus suaque ante insignia sumat) ${ }^{56}$ Caligola, poi, che amava abbigliarsi nelle maniere più strane e, spesso, anche non umane, se ne andava in giro plerumque vero aurea barba, fulmen tenens aut fuscinam aut caduceum deorum insignia ("con la barba dorata, oppure tenendo in mano un fulmine, un tridente, un caduceo, insignia degli dèi”), ${ }^{57}$ assimilando il proprio aspetto esteriore a quello di un dio di volta in volta diverso, Giove, Nettuno o Mercurio. Proprio a tali tratti distintivi, perciò, doveva prestare particolare attenzione l'artista che doveva costruire delle immagini il cui oggetto fosse riconoscibile. Alcuni avevano una storia mitica e spesso la loro presenza presupponeva un evento leggendario alla loro origine. In questo caso si può parlare di insignia a carattere narrativo ed esempi erano l'alloro e la lira per Phoebus ${ }^{58}$ la pelle di leone per Eracle,${ }^{59}$ l' ulivo e l'ègida con la Gorgoneion per Minerva ${ }^{60}$ etc. Altri rimandavano a funzioni / officia della divinità: in questo caso si può parlare di insignia funzionali, ovvero di oggetti che esprimevano il campo d'azione e la sfera di influenza di un nume; esempi erano la lancia per Marte, i grappoli d'uva per Bacco, i calzari e il copricapo alati per Mercurio etc. Tuttavia a volte i due tipi di insignia potevano coincidere: un dio, infatti, si poteva servire di un insigne funzionale per compiere un' impresa mitica: solo per citare qualche esempio, la clava venne usata da Eracle per compiere alcune azioni eroiche come l'uccisione di Caco, così come l'arco, tradizionale insigne funzionale di Diana, venne usato dalla dea nell' uccisione di alcuni mortali macchiatisi di tracotanza, tra cui la stirpe delle Niobidi. Un ulteriore esempio dell' importanza che tali attributi assumevano nell' identificazione degli dèi ci è offerta da Ovidio che in maniera ironica prova a mescolare gli insignia divini:

"Che accadrebbe se Venere strappasse via le armi alla bionda Minerva, o se la bionda Minerva agitasse al vento le fiaccole ardenti? Chi potrebbe accettare che Cerere sia la regina delle selve montane e che i campi vengano coltivati agli ordini della vergine con la

55. Luc., Rer. nat. II 606-609. Cf. Bettini, 2016, p. 172.

56. Ov., Met. III 283-285; Val. Fla., Arg. VI 52-53: cuncta phalanx insigne Iovis caelataque gestat / tegmina dispersos trifidis ardoribus ignes.

57. Bettini, 2015a, p. 176. Suet., Calig. 52.

58. Verg., Ecl. III 62; Hyg., Fab. 202; Ov., Met. I 557-567.

59. Hes., Theog. 326-332; Pind., Isthm. VI 47-48.

60. Hdt., Hist. VIII 55; Apollod., Bibl. XIV 1. 
faretra? Chi potrebbe fornire a Febo, che si distingue per la sua chioma, un' aguzza lancia mentre Marte fa risuonare le corde della lira aonia?".61

Evidentemente si potrà dire con una certa sicurezza che, dal momento che i Romani basavano la loro conoscenza delle divinità sugli insignia, è chiaro che se essi venissero capovolti o mescolati ciò comporterebbe una perdita della loro cognitio, della loro "riconoscibilità" come singole personalità del popolo divino. ${ }^{62}$ Tale importanza può infine essere evidenziata anche in una divertente Elegia dei Priapea nella quale il povero Priapo, per difendere l' uso della sua "arma" e dunque del suo officium, nomina gli altri dèi in base ai loro attributi visuali, insigna del loro potere: come Giove non si vergogna ma anzi mostra apertamente il suo fulmen, come Nettuno non nasconde i suoi fuscina o Marte la sua ensis, come Atena non si vergogna della sua hasta, Febo e la sorella Diana delle loro pharetrae e sagittae, l'Alcide della sua clava, Bacco del suo thyrsus e Amore della sua faces, perché Priapo dovrebbe nascondere e non mostrare apertamente la sua mentula? In fondo, è un' arma come un' altra, in fondo è un insigne anch'esso al pari degli altri insignia divini. ${ }^{63}$

Gli insignia, innanzitutto, non erano designatori rigidi; molte divinità, infatti, erano caratterizzate dagli stessi attributi visuali (Phoebus e Diana, Mars, Minerva e Bellona, Ianus e Portunus solo per citare alcuni esempi) e un solo oggetto, in diversi casi, non permetteva l'immediata identificazione di un dio. Le divinità erano puissances cangianti e polisemiche e la loro riconoscibilità necessitava di uno sforzo di comprensione dei loro attributi a seconda della funzione e dei contesti in cui erano istallati; lo spettatore, infatti, si trovava di fronte a più segni e solo con la loro interpretazione complessiva poteva arrivare all' identificazione più o meno inequivocabile del nume preso in esame. Gli insignia, perciò, erano anche designatori relazionali che legavano più dèi in una fitta rete di nessi contestuali e visivi. Gli insignia inoltre

61. Ov., Ars Amandi I 1, 7: quid, si praeripiat flavae Venus arma Minervae, / ventilet accensas flava Minerva faces, / quis probet in silvis Cererem regnare iugosis, / lege pharetratae Virginis arva coli?/ Crinibus insignem quis acuta cuspide Phoebum / instruat, Aoniam Marte movente lyram?/ Sunt tibi magna, puer, nimiumque potentia regna; / cur opus adfectas, ambitiose, novum? (trad. L. Canali 1990).

62. Bettini, 2016, p. 175.

63. Priapea, IX: Cur obscena mihi pars sit sine veste, requiris: / quaere, tegat nullus cur sua tela deus. / Fulmen habet mundi dominus, tenet illud aperte; / nec datur aequoreo fuscina tecta deo. / Nec Mavors illum per quem valet, / occulit ensem; / nec latet in tepido Palladis hasta sinu. / Num pudet auratas Phoebum portare sagittas? / Clamne, solet pharetram ferre Diana suam? / Num tegit Alcides nodosae robora clavae? / Sub tunica virgam num deus ales habet? / Quis Bacchum gracili vestem praetendere thyrso, / quis te celata cum face vidit, Amor? / Nec mihi sit crimen quod mentula semper aperta est: / hoc mihi si telum desit, inermis ero. 
agivano come marche di riconoscibilità non solo all' interno del proprio pantheon di riferimento, ma potevano servire parallelamente al meccanismo di contatto e dialogo tra sistemi religiosi, nella ricostruzione e identificazione personale di divinità appartenenti ad una cultura diversa (secondo il cosiddetto meccanismo dell' interpretatio); in altre parole, dovendo adattare e riconfigurare una divinità "straniera" in una divinità pienamente comprensibile e "conoscibile" al proprio pantheon, si potevano utilizzare gli attributi rituali che un dio o una dea possedeva in un sistema cultuale "altro" e appropriarsene stabilendo un legame con gli insignia di altri dèi o dee ben affermate nell' ambito della proprio sistema cultuale. La divinità "straniera", così, aveva più possibilità di essere "tradotta" e "riconosciuta" (per la vis e facultas necessaria ad una corretta invocazione) pur mantenendo degli elementi eterogenei, come si può rilevare in questo passo delle Historiae di Tacito relativo a Serapis:

"Per quel che riguarda il dio [Serapide], molti ritengono che sia Esculapio, perché cura le malattie corporali; secondo altri è Osiride, antichissima divinità di quei popoli; per la maggior parte si tratta invece di Giove, perché è signore di tutte le cose; molti però congetturano che sia il padre Dite, sia sulla base degli attributi che apertamente mostra, sia ricorrendo a tortuose elucubrazioni”. ${ }^{64}$

L' identificazione della divinità avveniva in base a delle congetture (coniectant), attraverso un procedimento di appropriazione e esclusione in cui a guidare l' interpretazione erano gli elementi di "rassomiglianza" che il dio condivideva tra più sistemi cultuali: in questo caso il campo d'azione, la tradizione religiosa, la potenza e gli attributi tipici del dio. Le immagini erano così commentate (oltre che contemplate) e sollecitate secondo un'estrema varietà di modalità operatorie concrete, configurandosi come un insieme complesso di "segni" che erano altrettanti referenti culturali e cultuali specifici. Tale procedimento è ben attestato anche in ambito figurativo, dal momento che anche i circuiti di diffusione delle immagini ponevano in questione $\mathrm{i}$ referenti stilistici e iconografici con le loro modifiche e mutazioni; caso peculiare in tal senso è rappresentato da una divinità polimorfa e soggetta a numerose interpretationes come Iside. Iside, o piuttosto le sue immagini e con esse i suoi insignia subirono multiformi identificazioni non solo nel tempo, ma anche nello spazio in cui vennero di volta in volta impiantati. La polisemia dei referenti iconografici (equivalenti dei

64. Tac., Hist. IV 84, 5: deum ipsum multi Aesculapium, quod medeatur aegris corporibus, quidam Osirin, antiquissimum illis gentibus numen, plerique Iovem ut rerum omnium potentem, plurimi Ditem patrem insignibus, quae in ipso manifesta, aut per ambages coniectant (trad. M. Bettini 2015b, p. 42). Cf. Bettini 2015b, p. 42. 
suoi numerosi epiteti ed epiclesi) rendeva possibile, per lo spettatore greco o romano, una comprensione immediata delle differenti facies della divinità egiziana e la cognitio delle sue prerogative in un gioco di identità multiple, poiché di volta in volta la sua immagine presentava delle caratteristiche dell' "altro" (l'egiziano, il greco, il punico etc.) e dell' identico, che poteva essere quello di Diana, Cerere, Fortuna etc., in un processo in cui diversi repertori iconografici e di conseguenza religiosi potevano essere scelti, combinati e scartati secondo necessità e scelte personali ${ }^{65}$ (figg. 1 e 2).

\section{QUANDO GLI ATTRIBUTI TRAVALICANO IL SIGNUM}

Vi erano alcuni casi, tuttavia, in cui il potere semiotico degli insignia travalicava il resto dell' immagine: in altri termini, non serviva più che un determinato oggetto fosse ricollegato ad un "corpo divino" specifico, dal momento che esso ne costituiva l' immagine totale, guidando così l' identificazione del dio. Un primo esempio in tal senso, già evidenziato da Bettini, è rappresentato dagli insignia funzionali di Marte conservati nella Regia e nella Curia Saliorum. Le hastae Martis, lance profetiche alle quali si accordò sempre la più grande venerazione, talvolta si scuotevano da sole, producendo apparentemente un rumore che annunciava eventi pericolosi (eventi che gli storici registrano con la frase hastae Martis in Regia sponte sua motae sunt); ${ }^{66}$ sulla sommità del Palatino invece, oltre al lituus di Romolo, erano conservati anche i due scudi o ancilia che si agitavano in ugual modo per profetizzare momenti particolarmente critici. In questi casi, la lancia e gli scudi, normalmente insignia di Mars, erano direttamente identificati con lo stesso dio: Plutarco, nella vita di Romolo, affermava che la lancia nella Regia era chiamata "A $\rho \uparrow \varsigma^{67}$ in un rapporto di immediata identificazione tra il dio e un suo attributo. Alcuni primitivisti (Herbert Jennings Rose in particolare $)^{68}$ hanno voluto vedere in questi episodi la fantasiosa quanto erronea presenza della forma più elementare del sacro, una "forza" diffusa o meglio dispersa che prima di qualsiasi tipo di rappresentazione religiosa, prima della nozione di dio personale, si "disperdeva" anche in numerosi supporti materiali. La lancia e gli scudi di Marte erano usati perciò come un ottimo esempio per provare che i Romani

65. Bricault e Prescendi in Bonnet, Pirenne-Delforge e Praet, 2009, pp. 63-79; Bricault e Versluys, 2012, pp. 1-23.

66. Gell., NA IV 6, 1-2; Liv., XXIV 10; XL 19; XXII 1, 11; cf. Serv., Ad Aen. VIII 3.

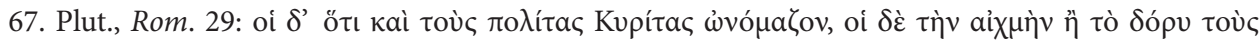

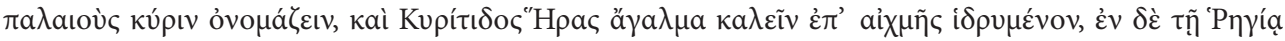

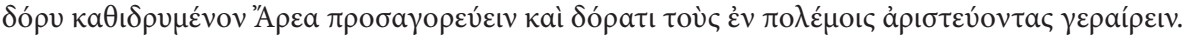

68. Rose, 1949, pp. 21-22. 
avessero creduto alle origini del pensiero sacro ad una forza astratta che si sarebbe evoluta nella forma "personificata” del dio Marte. Georges Dumézil, ponendosi agli antipodi del pensiero primitivista, coglieva invece un aspetto interessante: ${ }^{69}$ i Romani, sin dall'epoca arcaica, avrebbero avuto ben presente Marte in quanto divinità "personificata", il suo officium e gli insignia legati ad esso; tuttavia, dal momento che tali oggetti permettevano la riconoscibilità di un dio, anche la loro presenza isolata poteva essere determinante nella sua caratterizzazione visuale. Essi, da soli, "facevano" il dio, ne manifestavano la praesentia, l'azione efficace che in questo caso riguardava il monito di un pericolo incombente. ${ }^{70}$

Un altro esempio significativo è rappresentato dal racconto ovidiano della metamorfosi del dio Aesculapius nel momento in cui deve essere trasportato dagli ambasciatori come nume tutelare a Roma dalla città di Epidauro (per allontanare dall' urbs una pestilenza verificatasi nel 293-292 a.C.). Il dio appare in sogno al Genius Romanus con lo stesso aspetto che aveva nel tempio (visus qualis in aede esse solet) $:^{71}$

“Tenendo nella sinistra un rozzo bastone, con la destra si lisciava la lunga e folta barba e dal placido petto emetteva queste parole: "Non temere, verrò, e lascerò il mio simulacro. Tu guarda soltanto questo serpente che con le sue spire sta avvolto intorno al mio bastone; osservalo bene per ricordare com'è. In questo serpente io mi trasformerò ma sarò più grande e avrò quell' imponenza che si addice ad una divinità quando si trasforma." Subito disparve con la voce il dio, con la voce e il dio il sonno, e alla fuga del sonno seguì il giorno che infonde la vita. L'aurora aveva volto in fuga i fuochi delle stelle: i dignitari, incerti sul da farsi, si radunano nel tempio oracolare e pregano il dio, chiesto dai Romani, d' indicare lui stesso con un segno divino in quale luogo volesse vivere. Non hanno ancora finito, che il dio in forma di serpente irto di grandi creste d'oro, si preannuncia con sibili e, giunto lì, fa tremare la propria statua, gli altari e le porte, il pavimento di marmo e il soffitto rivestito d'oro; poi si ferma al centro del tempio, sollevandosi con tutto il petto, e volge intorno gli occhi che lampeggiano di fuoco" ${ }^{72}$

69. Dumézil, 1977, pp. 35-38.

70. Bettini, 2016, p. 176.

71. Ov., Met. XV 650-651.

72. Ov., Met. XV 655-674: [..] baculumque tenens agreste sinistra / caesariem longae dextra deducere barbae / et placido tales emittere pectore voces: / 'pone metus! veniam simulacraque nostra relinquam. / hunc modo serpentem, / baculum qui nexibus ambit, / perspice et usque nota visu, ut cognoscere possis! / vertar in hunc: sed maior ero tantusque videbor, / in quantum verti caelestia corpora debent.' / extemplo cum voce deus, cum voce deoque / somnus abit, somnique fugam lux alma secuta est. / postera sidereos aurora fugaverat ignes: / incerti, quid agant, proceres ad templa petiti / conveniunt operosa dei, quaque ipse morari / sede velit, signis caelestibus indicet, orant. / vix bene desierant, cum cristis aureus altis/ in serpente deus praenuntia sibila misit / adventuque suo signumque arasque foresque / marmoreumque solum fasti- 
Il nume stesso annuncia ai legati che si "trasformerà" in quello che era un suo elemento figurativo, proprio quel serpente che era avvolto intorno al bastone con cui veniva frequentemente designato iconograficamente; ciò fa sì che i dignitari lo riconoscano immediatamente come divino, giacché, come insigne, il serpente "fa" il dio. Deus en, deus en! grida il sacerdote all' apparizione del dio, alla rivelazione del suo numen (numen visum, v. 680) e alla protezione che ha accordato (adnuit) al popolo romano. Finalmente riconosciuto come tale, il dio agita le creste ed emette sibili, gira il vultus per osservare i suoi antichi altari e si accomoda sulla nave romana, rivelando ancora una volta lo statuto divino grazie al suo peso superiore. I Romani conoscevano benissimo l' officium di Asclepio e gli insignia legati ad esso; anche la loro presenza isolata, dunque, era determinante nella caratterizzazione visuale di un personaggio divino giacché dimostravano la sua azione efficace che, in questo caso, era quella di protezione e purificazione accordata alla città di Roma. ${ }^{73}$

In alcuni episodi, infine, gli insignia si potevano autonomizzare dal dio che li "indossava" diventando elementi narrativi a tutti gli effetti; essi si staccavano dal nume e interpretati insieme, nella loro carrellata complessiva, offrivano l'identificazione della divinità in questione senza che essa venisse nominata. Questo, ad esempio, è ciò che succede nelle pagine del IV libro delle Metamorfosi di Ovidio quando il dio Dioniso si manifesta attraverso i suoi insignia. Nel momento in cui le Miniadi, figlie del re Orcomeno in Beozia, si ostinavano a non cedere al culto orgiastico del dio onorando Minerva: "a un tratto timpani invisibili strepitarono con suono sordo, echeggiarono flauti a becco curvo e tintinnarono bronzi in un profumo di mirra e croco; e accadde un fatto incredibile: i telai cominciano a germogliare, le stoffe appese a mettere fronde in sembianza d'edera; parte si trasformò in viti e quelli che erano poco fa fili si mutarono in tralci; dagli orditi spuntarono pampini e la porpora usò il suo pigmento per dipingere l'uva" (trad. P. B. Marzolla, 2015). ${ }^{74}$ In questo episodio gli insignia (i pampini, i timpani e i flauti, l'edera, i grappoli d' uva) diventano oggetti potenti a tutti gli effetti, conducono e diventano protagonisti della narrazione mitica e interpretati insieme offrono l'identificazione di Dioniso / Bacco. La medesima

giaque aurea movit / pectoribusque tenus media sublimis in aede / constitit atque oculos circumtulit igne micantes (trad. P. B. Marzolla, 2015).

73. Sull'associazione tra Aesculapius e il serpente e le sue funzioni purificatrici Ogden, 2013, pp. 310316.

74. Ov., Met. IV 390-398: tympana cum subito non adparentia raucis / obstrepuere sonis, et adunco tibia cornu / tinnulaque aera sonant; redolent murraeque crocique, / resque fide maior, coepere virescere telae / inque hederae faciem pendens frondescere vestis; / pars abit in vites, et quae modo fila fuerunt, / palmite mutantur; de stamine pampinus exit; / purpura fulgorem pictis adcommodat uvis. 
predominanza dell' insigne che "spicca" nell' interpretazione del dio può essere ravvisata anche in un celebre affresco dalla "Casa del Centenario" a Pompei, "Bacco e il Vesuvio": il grappolo d' uva, tradizionale attributo iconografico del nume, viene direttamente identificato con lui, "realizza" e mette in scena da solo la divinità (fig. 3). ${ }^{75}$

\section{CoESISTENZA E SOVRABBONDANZA DI INSIGNIA}

A questo punto si potranno comprendere anche i casi di coesistenza di immagini e insignia multipli che caratterizzano la rappresentazione figurativa di alcune divinità, come nel caso di Juppiter Optimus Maximus Dolichenus, il cui culto si sviluppò in Siria a partire dal II sec. d.C. Originario di Doliche, capitale della Commagene (annessa alla provincia di Syria a partire dal 72 d.C.), il Dolichenus era rappresentato tradizionalmente insieme alla sua paredra Iuno regina come un imperator romano con il suo famoso paludamentum; esso rientrava nella tipologia di un Wettergott e si avvicinava a Iuppiter Optimus Maximus Capitolinus nella specificità di essere un dio chiamato a presiedere all'eternità ed alla stabilità del mondo del quale era conservator. Un dio omnipotens e aeternus dunque, che poteva essere invocato pro salute imperii Romani ${ }^{76}$ L'esempio emblematico in tal senso è quello dei triangoli dolicheni, placche bronzee che dovevano mettere in scena la teologia più o meno complessa del nume (fig. 4); il fatto di apparire come un dio caratterizzato da forza guerriera e da evidente marzialità ha permesso che intorno a lui si disponessero le rappresentazioni figurative e gli insignia di Hercules, Mars e Minerva, nonché l'immagine della Victoria. Ma al Dolichenus si accompagnavano anche Apollo, Asklepios/Aesculapius ed Hygieia/Salus che mettevano in scena le sue capacità di protezione e la sua qualità intramondana di conservator. Per analizzare tali complessi processi di circolazione di motivi cultuali bisogna sicuramente lasciare da parte il termine controverso e particolarmente inadeguato di sincretismo religioso, quelli di assimilazione, giustapposizione, associazione, sovrapposizione di immagini (concetti cari al pensiero primitivista, evoluzionista e "psicologista" del XIX-XX secolo), così come quello di "pagan monotheism", "megatheism" o di "filosofizzazione" dei sistemi religiosi in senso neoplatonico. ${ }^{77}$ Secondo questi approcci, gli dèi pagani avrebbero sempre avuto il potere, quando un culto locale attribuiva loro un rilievo particolare, di soggiogare, "inglobare" altre divinità e concentrare le loro proprietà in se stessi; il loro potere

75. Bettini, 2016, p. 177.

76. Sanzi, 2013, pp. 64-65.

77. Usener, 1896; Cumont, 1906; Otto, 1917; Dodds, 1965; Fowler, 1971; Athanassiadi, 2010; Mitchell e Van Nuffelen, 2010; Chaniotis in Mitchell e Van Nuffelen, 2010. 
diventava così universale, capace di un'estensione infinita. Ciò avrebbe aperto la strada a fusioni enoteistiche e panteistiche; quindi, a un processo "diffusionista" di evoluzione morale dell'Occidente e al successo del monoteismo cristiano. L'approccio storiografico e ritualista, invece, mostra come di fronte a tali pregiudizi è indispensabile un rinnovato approccio comparatistico (in termini di religioni in contatto piuttosto che in termini di diffusionismo e conversione) che metta in luce le strategie religiose degli "individual human agents" in situazioni cultuali concrete. ${ }^{78} \mathrm{Di}$ fronte a questa pluralità di referenti visuali, si dovrà pensare perciò alla produttività delle religioni antiche attraverso la coesistenza e convivenza di immagini polisemiche, in un meccanismo che potremmo definire di super interpretatio iconografica, attraverso lo scambio e la scelta incessante di referenti iconografici utili per la "comprensione" di una determinata divinità.

Lo stesso procedimento, infine, potrà essere ravvisato anche a proposito di tutta quella serie sterminata di oggetti, spesso di piccole dimensioni, la cui diffusione in tutto l' impero si colloca tra I-III sec. d.C., che prendono il nome (erroneo) di signa panthea (in base alla definizione che ne dà Franz Cumont nel Daremberg-Saglio); ${ }^{79}$ per alcuni ignoriamo l'identificazione con una specifica divinità, configurandosi come un gruppo sovrabbondante di attributi divini del pantheon greco e romano (per un esempio, fig. 5). Tali oggetti costituiscono, in primo luogo, un esempio del trionfo semiotico degli insignia e dunque dei poteri divini a cui tali oggetti rimandavano. Nonostante per la maggior parte di essi non si conoscano né i realizzatori materiali né la destinazione pratica, si può ragionevolmente presupporre che tali oggetti mettessero in scena una rappresentazione parziale ed efficace del divino: parziale perché era chiaramente impossibile rappresentare la "totalità" degli dèi (come invece Franz Cumont tentava di dimostrare utilizzando in modo improprio l'aggettivo pantheum, interpretando il prefisso pan come "totale" e non in senso accrescitivo-intensivo della persona o cosa a cui il termine è attribuito); ${ }^{80}$ efficace perché attraverso tali rappre-

78. Wissowa, 1912; Rudhardt, 1992; Borgeaud, 2004; Scheid, 2011; Bonnet, Pirenne-Delforge e Praet, 2009; Rüpke, 2016.

79. DAGR, s.v. panthea signa, pp. 315-317: si prende l' immagine di un dio particolare, "que l'on considère comme possédant les pouvoirs du panthéon tout entier"; per rappresentare ciò, gli si attribuiscono le caratteristiche "d' une foule de dieux divers" aumentando il numero, fino a cercare di esprimere "les fonctions innombrables de l'être universel" (!).

80. Hoenigswald, 1940; Chantraine, 1968, s.v. $\pi \tilde{a} \varsigma$, pp. 859-860; Leumann, 1950, pp. 98-107. I più antichi composti con tale prefisso hanno il senso di "complet, complètement": $\pi \alpha v \tilde{\eta} \mu \alpha \rho$ "tutta la giornata";

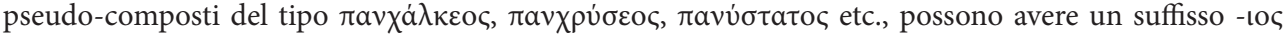

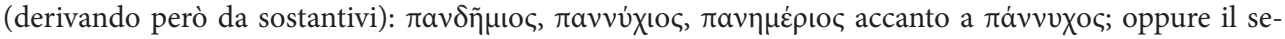

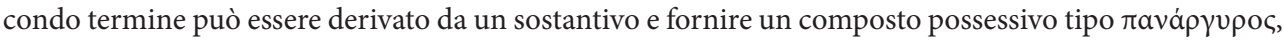


sentazioni il dedicante fabbricava un proprio universo di senso religioso e culturale, raffigurando attraverso gli insignia quelle divinità che gli erano particolarmente care. ${ }^{81}$ Secondo questa prospettiva, allora, il signum può essere pantheum solo se viene declinato con il significato di "del tutto divino, divino al massimo grado", proprio perché attraverso la creazione di questi oggetti si volevano raffigurare delle immagini che fossero "particolarmente divine" per l'attore religioso che le fabbricava e che dovevano colpire l'occhio a vederle tutte insieme, nella loro interpretazione complessiva. Un ultimo esempio, forse il più noto tra i signa panthea, sono le mani bronzee legate al culto di Sabazio, anch'esse rinvenute in varie zone dell' Impero Romano con un ventaglio cronologico abbastanza ampio (I a.C.-III d.C.). Divinità di probabile origine traco-frigia, Sabazio e il suo culto misterico-orgiastico si diffuse dapprima in Grecia (dove incontrò alcune resistenze) per poi approdare a Roma nel II sec. a.C., dove venne "interpretato" dapprima come Dioniso-Bacco, per poi accogliere le interpretationes di altre divinità legate al pantheon greco-romano. Nella definizione dell' iconografia di questa divinità, attestata dalle commedie di Aristofane sino alla fine del III-IV sec. d.C., si possono ravvisare due gruppi di esemplari: il primo consiste in rilievi iscritti in cui il dio viene rappresentato seduto o in piedi con in mano una lancia o uno scettro, ma anche un bastone, un cratere, un fulmine, un' aquila, una patera e un serpente (ad indicare le sue sfere di competenza marziali, cosmiche e ctonie che lo avvicinavano a Marte, Dioniso e Giove, vd. fig. 6); il secondo gruppo è costituito dalle figurine o dagli attributi del dio istallati in piccoli oggetti bronzei tra cui spiccano le mani votive che costituiscono il gruppo più significativo delle attestazioni iconografiche relative al suo culto $^{82}$ (tra cui alcuni splendidi esemplari pompeiani, vd. fig. 7). In particolare, tali oggetti erano caratterizzati da una profusione di attributi e di "esseri" divini distribuiti sulle due facce delle mani, ad indicare il triplo registro

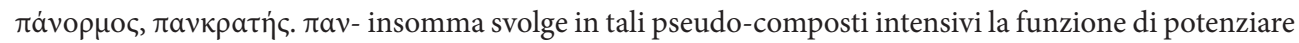
le qualità del sostantivo / aggettivo. In particolare si nota che il secondo membro può essere indifferentemente sostantivo o aggettivo ( $\pi \alpha v a ́ \rho \gamma v \rho \circ \varsigma / \pi a v \chi a ́ \lambda \kappa \varepsilon \circ \varsigma)$ e che il secondo termine, se è sostantivo, può

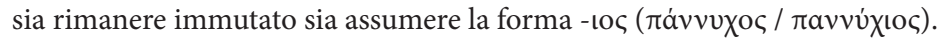

81. Ad esempio Fabio Pittore (16 HRR) secondo una notizia riportata anche da Dion. Hal., Ant. R. VII

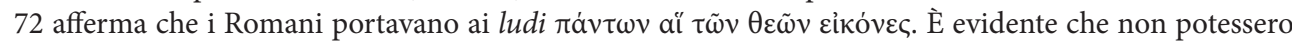
essere tutti e infatti di seguito vengono elencati: Zeus, Hera, Athena, Poseidone e i progenitori di questi, Kronos, Rhea, Themis etc., ovvero quelli che erano percepiti tra i più venerabili nel gruppo dei dodici dèi del pantheon greco e che quindi dovevano risaltare più degli altri; anche Varro, Rust. I 1, all' inizio dell'opera afferma l' intenzione di invocare "tutti" gli dèi, ovvero quelli che maxime agricularum duces sunt, quelle divinità (12 in tutto) che nell'orizzonte agreste di Varrone erano le più "tipiche".

82. Lane, 1980, pp. 13-15. 
vegetale, animale e astrale nel quale si esercitava l' influenza sovrana di Sabazio. ${ }^{83}$ In questo caso la dominazione, la potenza esercitata dal dio è esaltata proprio dall'accumulazione abbondante di elementi divini, racchiusi in maniera ordinata nella sua mano potente, che li domina e governa tutti. Questi oggetti dunque esprimono una teologia i cui elementi costitutivi formano un insieme certo eterogeneo, ma straordinariamente coerente; un insieme in cui si rivela, da un' immagine all'altra, una serie di analogie simboliche che vanno verso la medesima direzione globale, caratteristica proprio della magna dunamis del dio. Come per il Dolichenus, anche in questo caso il frazionamento progressivo del dio in più personalità divine mette in luce l' importanza assunta nella teologia romana dagli attributi visuali. Gli agenti religiosi, dovendo analizzare l'intervento delle divinità per trarne la corretta cognitio, estraevano dalla loro personalità, o meglio dalla loro azione, altre figure divine associate secondo la necessità e il contesto alla personalità e alla sfera d' influenza del dio di riferimento. Di conseguenza, per usare le parole di Scheid, "tutte queste figure divine e tutte queste strutture politeistiche collaborano nell'esprimere, in un contesto specifico, un unico concetto e un' unica azione: esse rappresentano un modo rituale di analizzare un principio divino". ${ }^{84}$

\section{Conclusioni}

Considerando il politeismo antico come una struttura dinamica e articolata, nella quale le molteplici potenze del mondo divino e umano erano collegate tra loro da una rete, flessibile e significativa, di relazioni, ci si è chiesti come i Romani "pensassero" i propri dèi attraverso alcune immagini culturali. Attraverso l'analisi del lessico romano relativo al mondo delle immagini si è evidenziato come la civitas immaginasse e costruisse l' esplicazione visuale delle divinità: i signa o gli insigna "evidenti" erano utilizzati per fabbricare, sia in senso intellettuale che concreto, le figurazioni del divino in modo che esse fossero identificabili come questo o quel personaggio della schiera innumerevole ed in perenne mutamento degli dèi; attraverso questi oggetti, infatti, essi rimarcavano la loro potenza, la loro vicinanza e la loro costante presenza nel mondo dei mortali. Analizzare la ricchezza dell' apparato figurativo di un dio, la fitta e mai monolitica relazione che i suoi attributi intessevano con altre divinità ci porta ad interrogarci sulla fluidità e porosità dei sistemi religiosi antichi. Di qui la necessità di prendere in considerazione non solo le singole figure divine che

83. Pailler in Bonnet, Pirenne-Delforge e Praet, 2009, pp. 257-291.

84. Scheid, 2011, p. 68. 
compongono i diversi pantheon e che si declinano variamente secondo il contesto, ma anche le immagini della società divina che gli antichi stessi hanno elaborato, $i$ racconti che mettono in scena la famiglia degli dèi, i culti rivolti ad una determinata configurazione di divinità: in una parola, le rappresentazioni antiche in cui più immediatamente si manifesta il divino al plurale. L'assenza di rappresentazioni dogmatiche, che potevano sorgere da testi rivelati o da un'autorità centrale, lasciava la porta aperta a tutti i rimodellamenti suscitati dalla diversità dei contesti e delle situazioni, soprattutto storiche: uno stesso attributo, perciò, istallandosi stabilmente su un signum o svincolandosi da esso, era così l'oggetto di elaborazioni e aggiustamenti permanenti, sotto forma di operazioni pragmatiche di "bricolage" (per citare Il pensiero selvaggio di Claude Levi-Strauss). Era anche attraverso l'ars, perciò, che gli uomini arrivavano a "conoscere" gli dèi e a dotarli di un' identità. 


\section{IMMAGINI}

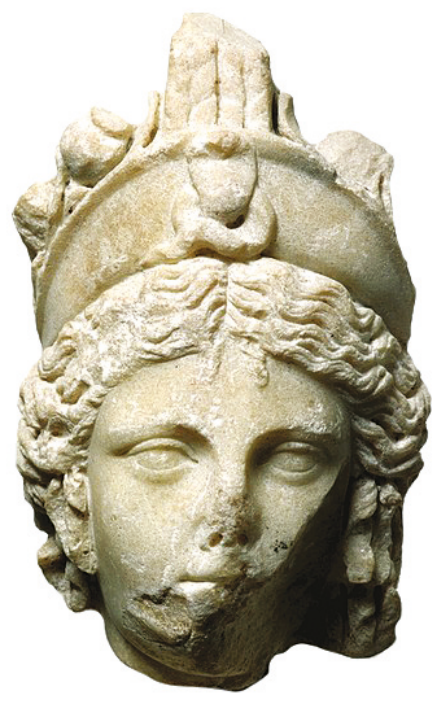

Fig. 1. Testa in marmo di Iside-Demetra rappresentata con diadema ornato da un ureus e sormontato da spighe e papaveri. II sec. d.C. Collezione Cappelli, Museo Nazionale Romano in Palazzo Altemps. Sala Isiaca, n. inv. 75065. (C) Fotosar - MIBAC Soprintendenza Speciale per i Beni Archeologici di Roma.

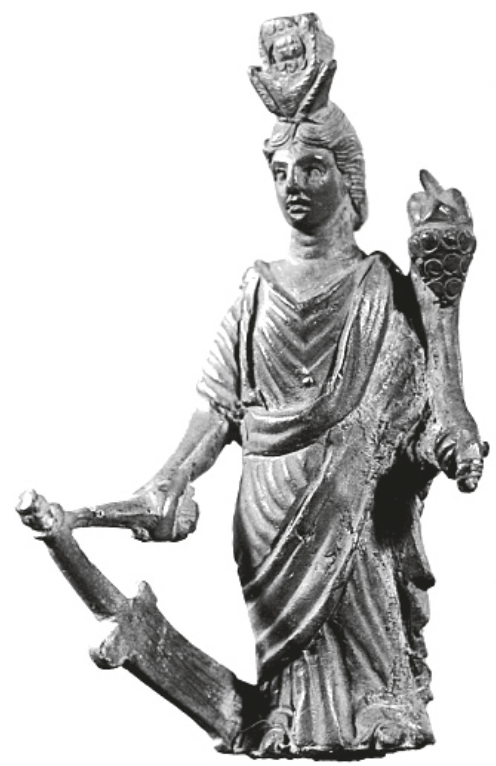

Fig. 2. Statuetta in bronzo (h. $9,8 \mathrm{~cm}$; p. $4,5 \mathrm{~cm}$ ) di IsideFortuna rappresentata con corno d'abbondanza nella mano sinistra e timone nella mano destra, insieme al tradizionale basileion. I sec. d.C. Da Ercolano, Casa del Colonnato Tuscanico. Soprintendenza Pompei, n. inv. 77559 B. Da V. Gasparini in Poole, 2016, p. 123. 
QUANDO GLI ATTRIBUTI TRAVALICANO IL SIGNUM.

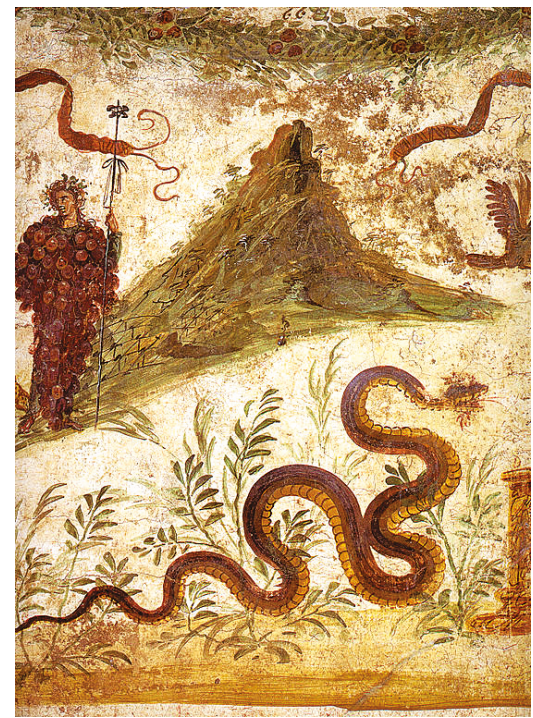

Fig. 3. Affresco raffigurante Bacco contornato da edera, un grappolo d' uva, un tirso e un serpente; sullo sfondo una montagna (il Vesuvio?) ricoperto di vigneti. I sec. d.C. Dal sacrarium della Casa del Centenario di Pompei. Museo

Archeologico Nazionale di Napoli, n. inv. 112286. Da Bettini, 2016, p. 177.

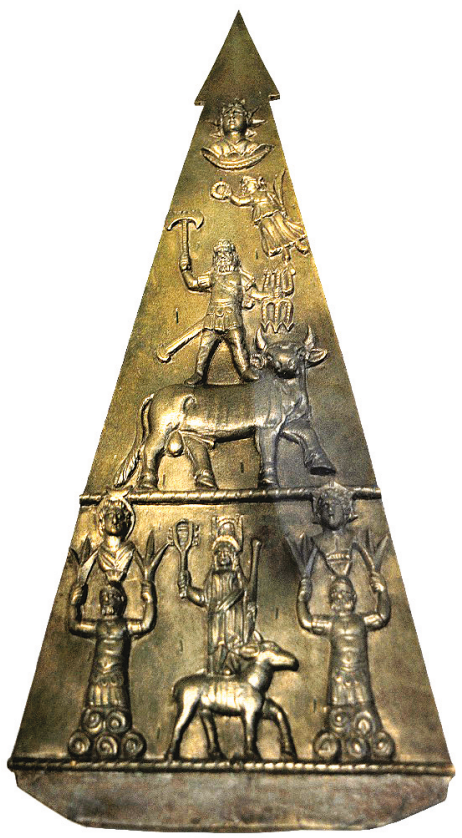

Fig. 4. Placca triangolare in bronzo fuso ( 49 × 19,5 x 3 $\mathrm{cm}$ ) raffigurante Sol, Iuppiter Dolichenus con berretto frigio, paludamentum e pantaloni orientali in piedi su un toro, una Vittoria e una divinità femminile su una cerva (probabilmente Iuno Dolichena); da entrambi i lati due Castores Dolicheni che sollevano dei fulmini, sormontati dai busti di Sole e Luna. 172 d.C. Da Heddernheim. Römermuseum, Osterburken. Da Merlat, 1951, p. 317. 


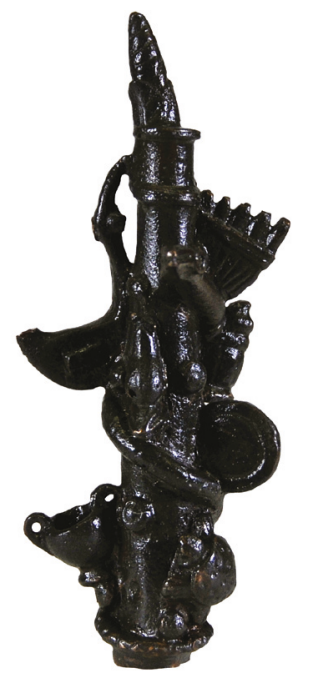

Fig. 5. Piccolo oggetto bronzeo $(10,7 \times 5,4 \times 3,6 \mathrm{~cm})$ raffigurante undici attributi divini disposti a spirale, dall' alto verso il basso, intorno ad un tronco: il fulmen di Giove, la syrinx di Pan, la lira di Apollo, la faretra di Diana, il cesto di Cerere, il timpano di Cibele, l' harpé di Saturno, il kantharos di Bacco, il delfino di Nettuno, la tartaruga di Mercurio, il serpente di Esculapio e Hygieia. I sec. d.C. Da Roma. Musée Saint Raymond, Toulouse, n. inv. 25625. (c) Ginevra Benedetti.

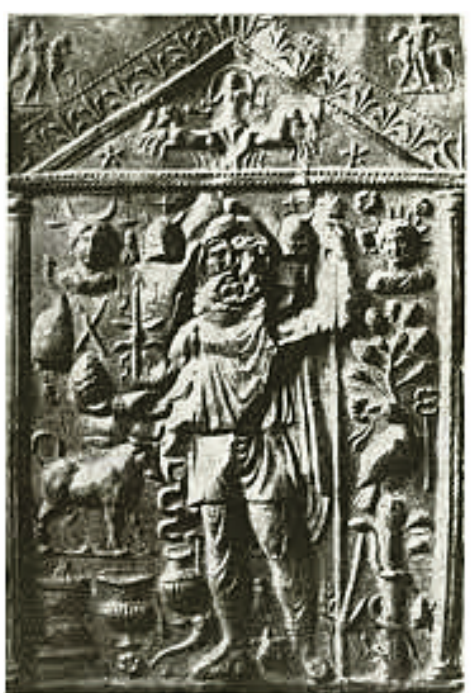

Fig. 6. Tavoletta in bronzo $(23,2$ x 15,6 x 0,05 cm) raffigurante

Sabazio in piedi iscritto in un' aedicula; nel timpano Helios in una quadriga e due stelle; fuori dal timpano, a destra e a sinistra, i due Dioscuri, Sole e Luna. Intorno a Sabazio si dispongono ventiquattro oggetti. II sec. d.C. Da Roma. National Museum of Copenaghen. Da CCIS II, pp. 3839 , t. 80. 
QUANDO GLI ATTRIBUTI TRAVALICANO IL SIGNUM.

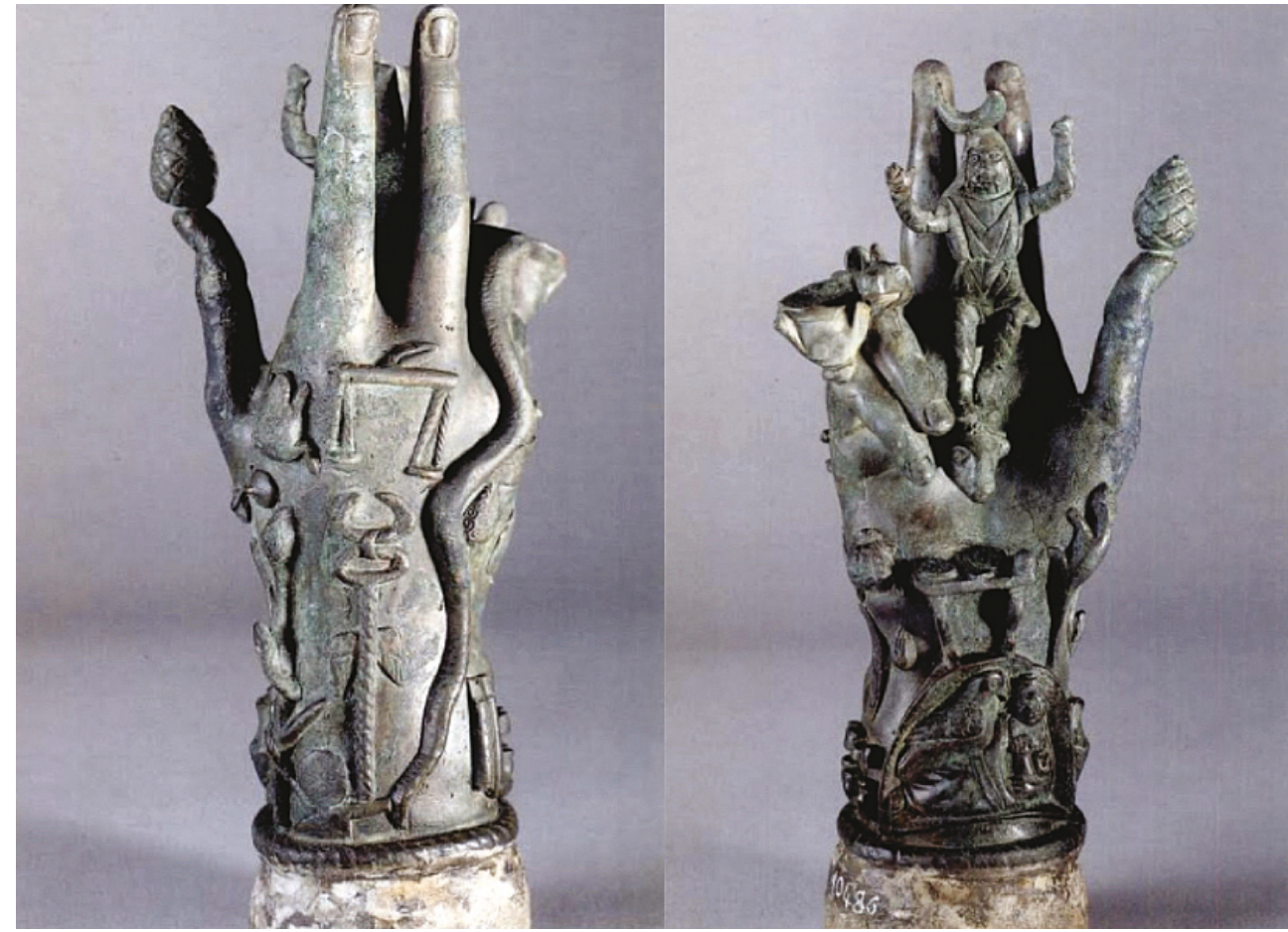

Fig. 7. Mano destra bronzea ritta e aperta $(18,7 \times 2,7 \times 5,5 \mathrm{~cm})$, l'anulare e il mignolo piegati, legata al culto di Sabazio. Sul polso figura una donna recumbente e allattante un bimbo e un personaggio maschile (identificato con Sabazio) vestito di corta tunica, calzoni e alti calzari, berretto conico con falde pendenti sulle spalle, sormontato da un crescente lunare. Sul palmo e sul dorso si dispongono venti oggetti. I sec. d.C. Da Pompei, Casa di Sestilius Pyrricus o Complesso dei Riti Magici, n. inv. 10486. Da Franchi dell'Orto, 1990, pp. 138-139. 


\section{Abbreviazioni}

CCIS: Lane, E.N. (1985). Corpus Cultus Iovis Sabazii. Leiden: Brill.

DAGR: Daremberg, C. e Saglio, E. (1969). Dictionnaire des Antiquités Greques et Romaines, Graz: Akademische Druck-u. Verlagsanstal.

DELL: Ernout, A. e Meillet, A. (1967). Dictionnaire étymologique de la langue latine. Paris: Klinksieck.

OLD: Glare, P.G.W. (1994). Oxford Latin Dictionary. Oxford: Oxford Scholarly Editions.

TLL: Thesaurus Linguae Latinae (1900-). Lipsiae: in aedibus B.G. Teubneri.

\section{Bibliografia}

Ando, C. (2010). Praesentia Numinis. Part 1: The visibility of Roman gods. Asdiwal, 5, pp. 45-73.

Athanassiadi, P. (2010). Vers la pensée unique. La montée de l'intolérance dans l'Antiquité tardive. Paris: Les Belles Lettres.

Belting, H. (1998). Image et culte. Une histoire de l' image avant l' histoire de l'art. Paris: Cerf (ed. francese dell'originale Bild und Kult. Eine Geschichte des Bildes vor dem Zeitalter der Kunst. München: Beck. 1990).

Belting, H. (2004). Pour une anthropologie des images. Paris: Gallimard (ed. francese dell'originale Bild-Anthropologie. Entwürfe für eine Bildwissenschaft, München: Fink. 2001).

Benveniste, E. (1932). Le sens du mot Kolossos et les noms grecs de la statue. RPhil SER, 36, pp. 118-135.

Bettinetti, S. (2001). La statua di culto nella pratica rituale greca. Bari: Levante Editori.

Bettini, M. (2000). Le orecchie di Hermes: studi di antropologia e letterature classiche. Torino: Einaudi.

Bettini, M. (2015a). Il dio elegante. Vertumno e la religione romana. Torino: Einaudi.

Bettini, M. (2015b). Dèi e uomini nella città. Antropologia, religione e cultura nella Roma antica. Roma: Carocci.

Bettini, M. (2016). Insignia. Identité et construction sémiotique de l'image divine à Rome. I Quaderni del Ramo d'Oro online, 6, pp. 162-180.

Bodiou, L., Frère, D. e Mehl, V. (eds.) (2006). L'expression des corps. Gestes, attitudes, regards dans l'iconographie antique. Rennes: Presses Universitaires de Rennes.

Bonnet, C., Pirenne-Delforge, V. e Praet, D. (2009). Les religions orientales dans le monde grec et romain : cent ans après Cumont (1906-2006) : bilan historique et historiographique. Colloque de Rome (16-18 novembre 2006). Rome: Institut historique belge de Rome.

Borgeaud, P. (2004). Aux origines de l' histoire des religions. Paris: Seuil.

Borgeaud, P. e Fabiano, D. (eds.) 2013. Perception et construction du divin dans l'Antiquité. Genève: Librairie Droz. 
Brachet, J.P. (1994). Esquisse d' une histoire de lat. Signum. Revue de Philologie, de Littérature et d'Histoire Anciennes, 68.1, pp. 33-50.

Bricault, L. e Versluys, M.J. (2012). Egyptian gods in the Hellenistic and Roman Mediterranean: Image and reality between local and global. Proceedings of the II International PhD workshop on Isis studies, Leiden University, January 26-2011. Suppl. to Mythos 3. Palermo: Salvatore Sciascia Editore.

Chantraine, P. (1968). Dictionnaire étymologique de la langue grecque: histoire des mots. Paris: Klincksieck.

Cumont, F. (1906). Les religions orientales dans le paganisme romain. Turnhout: Brepols $\mathrm{Pu}-$ blishers (ed. 2010).

Dasen, V. e Wilgaux, J. (eds.) (2008). Langages et métaphores du corps dans le monde antique. Rennes: Presses Universitaires de Rennes.

Dewailly, M. (2001). Image et religion. Méthodes et problématiques pour l'Antiquité gréco-romaine. Le rôle de l'image divine dans la définition de l'espace sacré. MEFRA, 113.1, pp. 175-400.

Dodds, E.R. (1965). Pagan and Christian in an age of anxiety. Some aspects of religious experience from Marcus Aurelius to Constantine. Cambridge: Cambridge University Press.

Dumézil, G. (1977). La religione romana arcaica. Miti, leggende e realtà. Milano: Rizzoli (ed. italiana dell'originale La religion romaine archaïque. Paris: Payot. 1966).

Elsner, J. (2007). Roman Eyes: Vision and Subjectivity in Art and Text. Princeton: Princeton University Press.

Estienne, S. (2001). Les “dévots” du Capitole. Le "culte des images" dans la Rome impériale, entre rites et superstition. MEFRA, 113.1, pp. 189-210.

Estienne, S. e Lissarague, F. (2005). Le corps des dieux dans les mondes grec et romain : bilan historiographique. DHA, Suppl. 14, pp. 19-29.

Estienne, S., Jaillard, D., Lubtchansky, N. e Pouzadoux, C. (eds.) (2008). Image et religion dans l'Antiquité gréco-romaine. Naples; Athènes: Centre Jean Bérard École française d'Athènes.

Estienne, S., Huet, V., Lissarrague, F. e Prost, F. (eds.) (2015). Figures de dieux. Construire le divin en images. Rennes: Presses Universitaires de Rennes.

Forcellini, E. (1965). Lexicon Totius Latinitatis. Bononiae: Forni.

Fowler, W.W. (1971). The religious experience of the Roman people, from the earliest times to the age of Augustus : the Gifford lectures for 1909-10 delivered in Edinburgh University. New York: Cooper Square.

Franchi dell' Orto, L. (ed.) (1990). Rediscovering Pompeii. Roma: L' Erma di Bretschneider.

Garelli, M.-H. e Visa-Ondarçuhu, V. (2008). Corps en jeu. De l'Antiquité à nos jours. Rennes: Presses Universitaires de Rennes.

Gladigow, B. (1985-1986). Präsenz der Bilder - Präsenz der Götter. Kultbilder und Bilder der Götter in der griechischen Religion. Visible Religion, 4-5, pp. 114-133.

Gladigow, B. (1990). Epiphanie, Statuette, Kultbild. Griechische Gottesvorstellungen im Wechsel von Kontext und Medium. Visible Religion, 7, pp. 98-121. 
Gladigow, B. (1994). Zur Ikonographie und Pragmatik römischer Kultbilder. In Keller e Staubach, 1994, pp. 9-24.

Gordon, R.L. (1979). The Real and the Imaginary: production and religion in the Graeco-Roman World. Art history, 2.1, pp. 5-34.

Hoenigswald, H.M. (1940). Pan Compounds in Early Greek. Language, 16, pp. 183-188.

Keller, H. e Staubach, N. (eds.) (1994). Iconologia sacra. Mythos, Bildkunst und Dichtung in der Religions- und Sozialgeschichte Alteuropas. Berlin: Walter de Gruyter.

Lane, E.N. (1980). Towards a definition of the iconography of Sabazius. Numen, 27.1, pp. 9-33.

Leumann, M. (1950). Homerische Wörter. Basel: F. Reinhardt.

Lewis-Short: Lewis, C.T. e Short, C. (1962). A latin dictionary. Oxford: Clarendon Press.

Linant de Bellefonds, P. (2004). Rites et activités relatifs aux images de culte. ThesCRA, III.5, pp. 417-507.

Malamoud, C. e Vernant, J.-P. (eds.) (1986). Corps des dieux. Paris: Gallimard (ed. 2003).

Merlat, P. (1951). Répertoire des inscriptions et monuments figurés du culte de Jupiter Dolichenus. Paris e Rennes: Librairie Orientaliste Paul Geuthner e Librairie les Nourritures Terrestres.

Mitchell, S. e Van Nuffelen, P. (2010). One God: Pagan Monotheism in the Roman Empire. Cambridge: Cambridge University Press.

Mylonopoulos, J. (2010). Divine Images and Human Imaginations in Ancient Greece and Rome. Leyden e Boston: Brill.

Ogden, D. (2013). Drakōn. Dragon myth and serpent cult in the Greek and Roman world. Oxford: Oxford University Press.

Otto, R. (1917). Il sacro : l'irrazionale nella idea del divino e la sua relazione al razionale. Milano: Gallone (ed. it. 1988).

Pirenne-Delforge, V. e Belayche, N. (2015). Fabriquer du divin. Constructions et ajustements de la représentation des dieux dans l'Antiquité. Liège: Presses universitaires de Liège.

Pironti, G. (2012). Poesia e religione: il pantheon ellenico tra invenzione e tradizione. In $\mathrm{La}$ grande storia. L'Antichità. Grecia. Volume sei: Mito e religione (pp. 42-53). Milano: RCS quotidiani.

Pironti, G. e Bonnet, C. (eds.) 2017. Les dieux d'Homère. Polythéisme et poésie en Grèce ancienne. Liège: Presses Universitaires de Liège.

Poole, F. (ed.) (2016). Il Nilo a Pompei. Visioni d'Egitto nel mondo romano. Catalogo della mostra (Torino, 5 marzo - 4 settembre 2016). Modena: Franco Cosimo Panini.

Prost, F. e Wilgaux, J. (dir.) 2006. Penser et représenter le corps dans l'Antiquité. Rennes.

Rose, H.J. (1949). Ancient Roman Religion. London: Hutchinsons University Library.

Rudhardt, J. (1992). De l'attitude des Grecs à l'égard des religions étrangères. Revue de l' histoire des religions, 209.3, pp. 219-238.

Rüpke, J. (2010). Representation or presence? Picturing the divine in Ancient Rome. Archiv für Religionsgeschichte, 12.1, pp. 181-196.

Rüpke, J. (2016). On Roman Religion: Lived Religion and the Individual in Ancient Rome. Ithaca; London: Cornell University Press. 
QUANDO GLI ATTRIBUTI TRAVALICANO IL SIGNUM.

Sanzi, E. (2013). Iuppiter Optimus Maximus Dolichenus. Un "culto orientale" fra tradizione e innovazione: riflessioni storico-religiose. Roma: Lithos.

Scheid, J. (2011). Quando fare è credere: i riti sacrificali dei Romani. Roma: Laterza.

Usener, H. (1896). I nomi degli dèi. Saggio di teoria della formazione dei concetti religiosi. Brescia: Morcelliana (ed. 2008).

Vernant, J.P. (1979a). Religion, histoire, raisons. Paris: Gallimard.

Vernant, J.P. (1979b). Naissance d' images. In Vernant, 1979a, pp. 105-137.

Vernant, J.P. (1990). Figuration et images. Mètis, 5, pp. 225-238.

Wissowa, G. (1912). Religion und Kultus der Römer. München: Beck. 